\title{
Quantum Physics With Ultrabroadband and Intense Terahertz Pulses
}

\author{
Alexej Pashkin, Friederike Junginger, Bernhard Mayer, Christian Schmidt, Olaf Schubert, \\ Daniele Brida, Rupert Huber, and Alfred Leitenstorfer
}

\begin{abstract}
We present recent advances in the generation of highly intense multiterahertz transients and their application to nonlinear spectroscopy of bulk semiconductors. An optimized scheme of parametric amplification results in broadband singleor few-cycle terahertz transients with peak electric fields up to 10 or $25 \mathrm{MV} / \mathrm{cm}$, respectively. Time-resolved four-wave mixing terahertz spectroscopy of InSb far away from the interband resonance demonstrates clear signatures of a nonperturbative regime of Rabi flopping. We qualitatively explain the observed behavior within a model of a quantum two-level system. In addition, we demonstrate the dynamical Franz-Keldysh effect in InP resolved on a subcycle timescale. The field-induced modulation of the interband optical absorption at the second harmonic of the driving terahertz field is observed in full agreement with theoretical predictions.
\end{abstract}

Index Terms-Electrooptic effects, four-wave mixing, nonlinear optics, semiconductor materials, terahertz technology, ultrafast optics.

\section{INTRODUCTION}

$\mathbf{S}$ EMICONDUCIORS represent a perfect model to investigate light-matter coupling in quantum many-body systems. Understanding this interaction in the limit of very intense electromagnetic fields is one of the most intriguing problems of modern optics and high-speed optoelectronics. Recent advances in high-field terahertz technology have opened up exciting new perspectives for studies of extremely nonlinear (NL) phenomena in semiconductors. Ultrashort timescales, inherent to terahertz transients, allow for applying electric fields, with amplitudes of multiple megavolt per centimeter, without irreversible breakdown of a sample. Another important advantage of terahertz technology is the possibility to generate phase-stable transients, and to perform field-resolved detection, which captures a profile of the electric field on a femtosecond timescale. Experiments on bulk semiconductors, performed using terahertz transients with peak fields up to $1 \mathrm{MV} / \mathrm{cm}$, have addressed a number of interesting phenomena such as intervalley scattering [1], ballistic transport [2], impact ionization [3], [4], and Rabi flopping of intersubband [5] or intraexcitonic [6] resonances.

The latest development of an ultraintense terahertz source with peak fields above $100 \mathrm{MV} / \mathrm{cm}$ paves the way toward studies of extremely NL response in semiconductors as well as in other condensed matter systems [7], [8]. Here, we present an overview of this high-field terahertz technology with a focus on recent advances in the generation of ultrabroadband singlecycle multiterahertz transients with peak electric fields above $10 \mathrm{MV} / \mathrm{cm}$. Then, we introduce two examples of experiments where these intense transients are exploited for off-resonant excitation of bulk semiconductors. In the first experiment, we study the time- and field-resolved terahertz four-wave mixing in bulk InSb using a phase-sensitive 2-D spectroscopy. The experimental results provide clear evidence for a nonperturbative response of the interband polarization. In the second experiment, we monitor the electroabsorption around the interband resonance of bulk InP induced by high-field multiterahertz transients via the dynamical Franz-Keldysh effect (DFKE). The phase stability of the terahertz pulses and the high temporal resolution of our experimental setup allow us to trace the modulation of the optical absorption on a subcycle timescale in contrast to previous studies [9], [10].

\section{Generation OF Ultrabroadband AND INTENSE TERAHERTZ. PULSES}

The basic process for the generation of terahertz transients is frequency mixing in a NL optical medium. Several groups have reported broadband terahertz radiation generated from laser-induced plasmas [11], [12]. This method relies on NL frequency conversion mediated by currents in the gas plasma and requires laser pulses with millijoule energies typically produced by Ti:sapphire-based amplifier systems operating at kilohertz repetition rates. An alternative way is difference frequency generation (DFG) which utilizes a second-order nonlinearity in optical crystals without inversion symmetry under suitable phase-matching conditions. When DFG occurs among different spectral components of the same optical laser pulse, the process is usually called optical rectification (OR). The lower order of the involved NL process allows efficient DFG using laser 
pulses in the nanojoule energy range [13]-[15]. The conversion efficiency of DFG/OR is expected to scale up linearly with the energy of the optical pulse. Thus, this method is also perfectly suitable for the generation of intense terahertz pulses. A rapid progress in high-power terahertz technology has been achieved recently by introducing a tilted-pulse-front excitation method which facilitates broadband phase matching in $\mathrm{LiNbO}_{3}$ crystals with a thickness of several millimeters [16]-[18]. The resulting terahertz transients contain only a single cycle of the field oscillation. Large optical nonlinearity of $\mathrm{LiNbO}_{3}$ makes OR particularly efficient: A photon conversion efficiency of $4.5 \%$ has been demonstrated using $20 \mathrm{~mJ}$ optical generation pulses [19]. Even higher efficiencies exceeding 100\% have been reported recently [20]. [21], however, the upper frequency of the spectrum is limited by the optical phonon absorption in $\mathrm{LiNbO}_{3}$ and reaches only $3 \mathrm{THz}$.

Multiterahertz transients with spectral components above $10 \mathrm{THz}$ can also be obtained by $\mathrm{DFG}$, provided that the optical pulses used for the generation possess a sufficient bandwidth. The birefringent crystals GaSe and $\mathrm{AgGaS}_{2}$, which enable a tunable phase matching at frequencies up to $70 \mathrm{THz}$, are most commonly used for this purpose [7], [14], [15], [22]. GaSe is an especially efficient material for the generation of intense multiterahertz pulses due to its wide transmission range and the exceptionally high second-order nonlinearity [23], [24]. It was shown that phase-matched OR of $0.6 \mathrm{~mJ}$ near-infrared pulses in $\mathrm{GaSe}$ is capable of generating few-cycle transients with peak electric fields above $1 \mathrm{MV} / \mathrm{cm}$ and carrier frequencies of $20 \mathrm{THz}$ [25]. However, the efficiency of OR rapidly decreases when the generation of multiterahertz transients at even higher frequencies is required since the DFG of high-frequency terahertz components can occur only by mixing the spectral wings of a generation pulse, as illustrated in Fig. 1(a). The resulting spectrum of the NL polarization produced by the OR process peaks at zero frequency and monotonically decays toward higher frequencies as shown in Fig. 1(b). Thus, a high photon-conversion efficiency in the multiterahertz range can hardly be achieved since the largest portion of the NL signal is generated at low terahertz frequencies. This problem is solved using two spectrally shifted near-infrared pulses [see Fig. 1(c)]: a relatively narrowband pump pulse centered at frequency $\omega_{2}$ and a broadband signal pulse at $\omega_{1}$. The DFG process can be viewed as an optical parametric amplification (OPA) process in which the amplification of the signal pulse occurs together with the generation of the multiterahertz idler component [26]. The spectrum of the idler schematically depicted in Fig. 1(d) peaks at the frequency $\omega_{2}-\omega_{1}$ corresponding to the spectral shift of the pump with respect to the signal. Thus, the photon conversion efficiency may be dramatically enhanced compared to the OR process [7]. More importantly, the bandwidth of the idler pulse is mainly defined by the bandwidth of the near-infrared signal pulse. Intense narrowband pump pulses can be used, thereby simplifying the generation scheme. Furthermore, the idler terahertz pulses generated via the OPA process are inherently phase-locked since a carrier-envelope phase offset of signal and pump pulses is canceled out during the DFG, if the generation of both pulses is seeded by the same laser source [7], [27], [28].
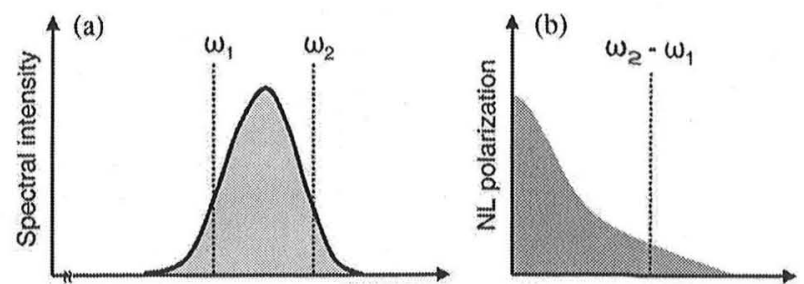

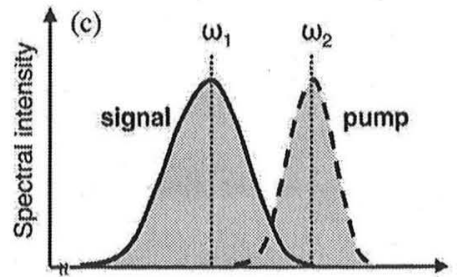

$\omega$

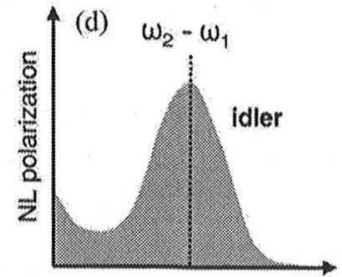

$\omega$
Fig. 1. Comparison between the OR and OPA processes. (a) Spectrum of a single ultrashort near-infrared laser pulse. (b) Corresponding spectrum of the terahertz NL polarization generated by DFG among spectral components within the single near-infrared pulse. (c) Spectra of the signal and pump pulses used for the OPA process. (d) Spectrum of NI. polarization of the generated terahertz idler pulse.

Here, we present a state-of-the-art ultrabroadband OPA-based source of phase-locked multiterahertz transients containing a single cycle of light. Our hybrid system combines the high stability and flexibility of fiber laser technology and high-power scalability of Ti:sapphire amplifiers [7]. A schematic view of the setup is shown in Fig. 2. A mode-locked Er:fiber master oscillator, working at a repetition rate of $49 \mathrm{MHz}$ and a center wavelength of $1.55 \mu \mathrm{m}$, seeds two parallel Er:fiber amplifier branches. The output of the first amplifier is frequency-doubled in a periodically poled $\mathrm{LiNbO}_{3}$ crystal and serves as seed for a high-power regenerative Ti:sapphire amplifier. The train of amplified 100-fs pulses with a repetition rate of $1 \mathrm{kHz}$ and an energy of $5 \mathrm{~mJ} / \mathrm{pulse}$ is split to pump two OPAs, which generate the near-infrared signal and pump pulses for the DFG process in a GaSe emitter crystal. The second Er:fiber amplifier branch generates the ultrashort gating pulses for the electrooptic detection: The broadband continuum is produced in a highly NL bulk silica fiber and compressed by a prism sequence resulting in pulses with a duration of $8 \mathrm{fs}$ centered at $1.2 \mu \mathrm{m}$ [29]. The gating pulses are delayed by a fast-scanning delay line and collinearly superimposed with the terahertz transient on the GaSe sensor crystal. The terahertz field-induced birefringence is detected using two balanced InGaAs photodiodes.

As discussed earlier, the generation of single-cycle multiterahertz transients requires near-infrared signal pulses with a large spectral bandwidth. Furthermore, the phase matching condition in GaSe has to be broadband enough to ensure efficient parametric amplification of all spectral components of the signal pulse. Recently, Junginger et al. have demonstrated that pumping at wavelengths longer than $1 \mu \mathrm{m}$ leads to a substantially increased bandwidth of the generated multiterahertzidler pulses [8]. Here, we consider an OPA process of type II in which the generated idler is polarized perpendicularly to the signal. Fig. 3 shows the calculated phase matching function $G(L)=|\sin (\Delta k L) /(\Delta k L)|$ for different internal propagation 


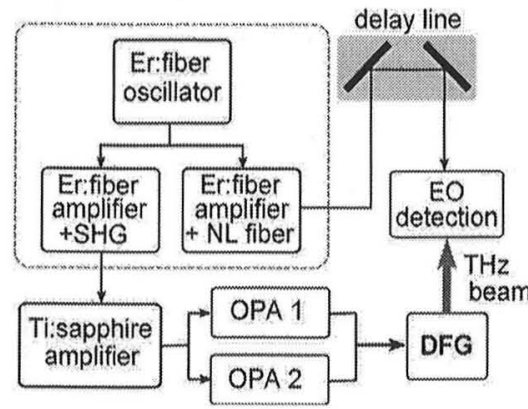

Fig. 2. Scheme of the hybrid laser system for the generation and electrooptic detection of intensive single-cycle terahertz transients. The output of the first Er: fiber amplifier is frequency-doubled via second-harmonic generation in order to match the wavelength of the seed pulses to the amplification band of Ti:sapphire. The second fiber amplifier generates a broad near-infrared continuum inside a highly NL fiber. OPA1 and OPA2 are double-stage optical parametric amplifiers; DFG is the difference frequency generation in a GaSe crystal; and EO detection is the electrooptic detection setup.

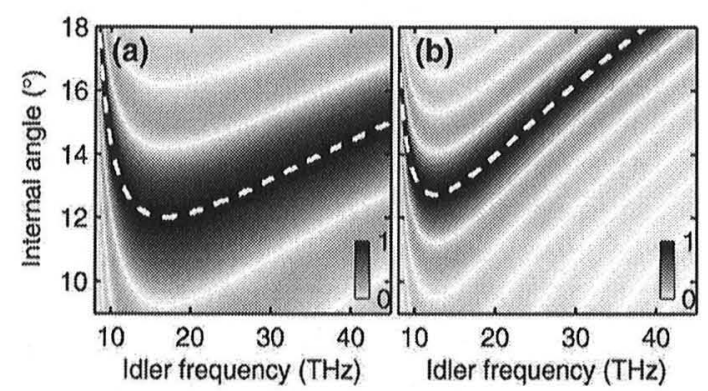

Fig. 3. Phase matching function $G(L)$ calculated for various internal propagation angles and the idler frequencies. The calculation is performed for type II parametric amplification in GaSe with a thickness of $L=90 \mu \mathrm{m}$. The central wavelength of the pump is set to (a) $1.26 \mu \mathrm{m}$ and (b) $0.8 \mu \mathrm{m}$. The dashed white curve marks the line of perfect phase matching.

angles and idler frequencies, where $\Delta k$ is the wave vector mismatch and $L$ is the thickness of the GaSe emitter crystal. We assume $L=90 \mu \mathrm{m}$ and two different pump wavelengths of $1.26 \mu \mathrm{m}$ [see Fig. 3(a)] and $0.8 \mu \mathrm{m}$ [see Fig. 3(b)]. The latter wavelength is typical for Ti:sapphire lasers. The optimal internal angle and the central frequency of the broadband phase matching are defined by the minimum of the dashed curves, which corresponds to $G(L)=1$. One can clearly see that the longer pump wavelength leads to a dramatic increase of the idler bandwidth, thanks to the matching of signal and idler group velocities [26]. For the case of the 1.26- $\mu \mathrm{m}$ pump wavelength, it is expected to exceed $10 \mathrm{THz}$ [see Fig. 3(a)]. The corresponding broadband signal pulse has to be centered around $1.38 \mu \mathrm{m}$.

The optimized OPA scheme for the generation of broadband near-infrared signal pulses introduced by Junginger et al. [8] is based on two amplification stages equipped with type I $\beta$-barium borate $(\mathrm{BBO})$ crystals. It enables the generation of broad spectra due to a small group-velocity mismatch (GVM). However, the tunability of the signal toward longer wavelengths is limited: Close to the degeneracy point, the broadband sig. nal and idler pulses start to overlap spectrally. Since both pulses have the same polarization in the case of the type $1 \mathrm{DFG}$ process, the signal pulse gets an admixture of the idler and, therefore, the generated terahertz radiation is not perfectly phase-stable.
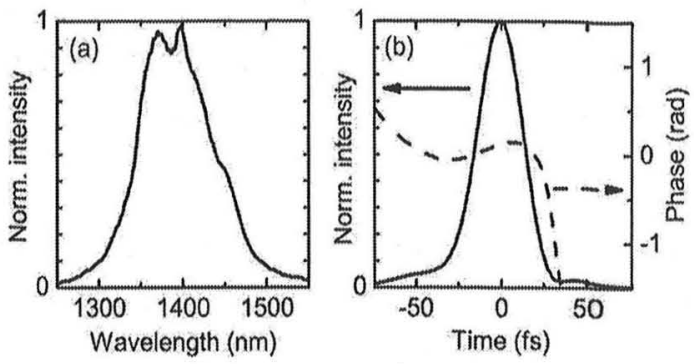

Fig. 4. Characteristics of the near-infrared pulse generated by the broadband OPA: (a) spectrum and (b) time-dependent intensity and phase profile of the pulse reconstructed from a FROG image.

The problem can be solved in the case of type II phase matching where signal and idler are polarized perpendicular to each other and can be easily separated. Thus, longer signal wavelengths required for the broadband phase matching in GaSe can be achieved. In the described scheme, the type II phase matching is not detrimental to the amplification bandwidth, thanks to the long wavelength of the idler and the similar group velocities in the terahertz range for ordinary and extraordinary propagation.

Fig. 4(a) shows the spectrum of the signal pulse generated by using a type II OPA scheme. The spectrum is centered at about $1380 \mathrm{~nm}$ and has a large full width at half maximum (FWHM) bandwidth of $97 \mathrm{~nm}$. Besides a good discrimination between the signal and idler, this scheme offers a very stable operation and it is weakly susceptible to thermal fluctuations and long-term drifts of the Ti:sapphire laser system. In contrast to type I phase matching, the signs of the GVM between signal and pump, and the GVM between idler and pump are opposite. Thus, signal and idler pulses move in opposite directions with respect to the pump pulse. As a result, both pulses tend to localize temporally around the pump pulse leading to their exponential amplification. A high-conversion efficiency causes a saturation of the amplification and a further back conversion via a sumfrequency process between signal and idler waves [30]. A typical signature of such behavior is the flattening in the center of the signal spectrum where the intensity is maximal as shown in Fig. 4(a). Another advantage of the type II OPA scheme in our setup is an extremely small amount of chirp in the amplified signal pulse. Fig. 4(b) shows the time profile of the intensity and phase of the signal pulse measured by a frequency-resolved optical gating (FROG) [31]. The temporal FWHM of the pulse is $31 \mathrm{fs}$ and the phase is almost flat indicating that the pulse duration is close to bandwidth-limited. This is in stark contrast to the previously reported type I OPA, where the signal pulse has a considerable chirp which has to be compensated by an external prism compressor [8]. The reason for this difference is the low dispersion added by propagation in $\mathrm{BBO}$ close to the zero-dispersion point and by the peculiar NL phase introduced during the amplification process [30].

We generate terahertz radiation by collinear DFG using signal pulses from OPA 1 and OPA 2 as shown in Fig. 2: The broadband pulse (see Fig. 4) serves as the signal, and the narrowband pulse from a tunable commercial OPA centered at $1.26 \mu \mathrm{m}$ plays the role of the pump. A GaSe emitter crystal is tilted with 

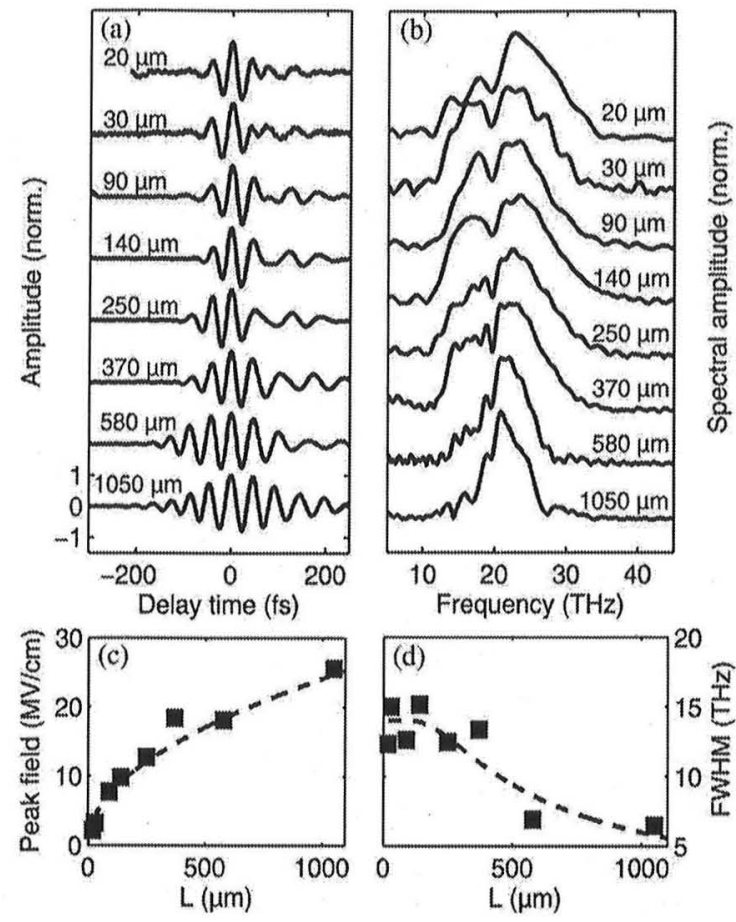

Fig. 5. (a) Real-time profile of the electric field of multiterahertz transients generated using DFG in GaSe emitters of different thicknesses measured by electrooptic detection in GaSe. (b) Normalized amplitude spectra of the transients. The arrow marks an air absorption band due to the bending mode of $\mathrm{CO}_{2}$ at $20 \mathrm{THz}$. (c) Peak electric field and (d) FWHM bandwidth of the multiterahertz transients as functions of the GaSe emitter thickness $L$. Dashed lines are guides to the eye.

respect to the incident beams in order to ensure the optimal internal propagation angle of $12^{\circ}$. The generation light remaining after the emitter crystal is blocked by a germanium wafer placed at the Brewster angle. Fig. 5(a) and (b) show the temporal profiles of the generated multiterahertz transients and their amplitude spectra, respectively, for different thicknesses $L$ of the GaSe emitter crystal. The broadband electrooptic detection is performed using a GaSe detector crystal with a thickness of $30 \mu \mathrm{m}$ (for $L \leq 90 \mu \mathrm{m}$ ) or $90 \mu \mathrm{m}$ (for $L \geq 140 \mu \mathrm{m}$ ). For $L \leq$ $90 \mu \mathrm{m}$, the FWHM of the intensity envelope of the transients is slightly below $50 \mathrm{fs}$ and, therefore, constitutes a single cycle of the terahertz oscillation period defined by the carrier frequency of $22 \mathrm{THz}$. We calibrate the peak electric field by measuring the average power of the terahertz beam using a standard broadband thermopile power meter (Melles Griot 13 PEM 001/J) and assuming a Gaussian spatial profile of the beam with a FWHM diameter of $55 \mu$ m estimated from the transmittance through a $50-\mu$ m pinhole. Fig. $5(\mathrm{c})$ shows the peak electric field of the transients as a function of the GaSe emitter thickness $L$. The field gradually increases with the increase of $L$ reaching $25 \mathrm{MV} / \mathrm{cm}$ for $L=1050 \mu \mathrm{m}$. This value is almost two times higher than in the report of Junginger et al. [8] demonstrating that the implementation of the type II OPA process for the signal generation permits to achieve high terahertz power for few-cycle transients. On the other hand, the peak electric field of the single-cycle pulses is slightly lower compared to the value of $12 \mathrm{MV} / \mathrm{cm}$ reported for the type I scheme [8].
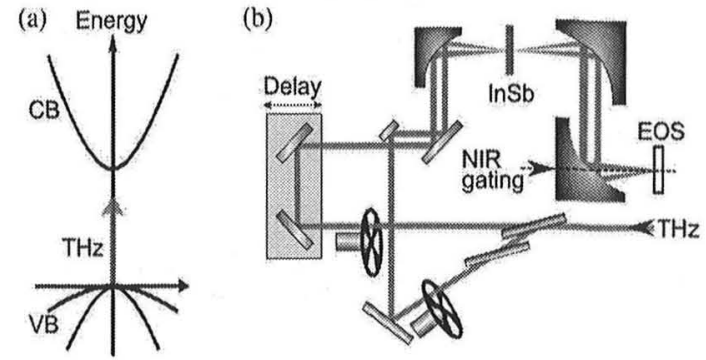

Fig. 6. (a) Off-resonant excitation of the transition between the valence and conduction bands of InSb. The arrow denotes the relative energy of a terahertz photon. (b) Scheme of the field-resolved FWM experiment: The terahertz beam is split into two branches, individually modulated by mechanical choppers. Terahertz pulses in one branch are delayed using a mechanical delay stage. The transmitted signals are detected by EOS.

Fig. 5(d) shows the FWHM bandwidth as a function of $L$. The single-cycle pulses obtained using thin emitters possess an FWHM bandwidth of about $15 \mathrm{THz}$, which is almost equal to the bandwidth of the signal pulse depicted in Fig. 4. Thus, when $L \leq 250 \mu \mathrm{m}$, the terahertz bandwidth is mainly limited by the spectral characteristics of the near-infrared generation pulses since the phase matching is very broadband. However, with increasing thickness of the emitter crystal, phase matching starts to decline over the full generation bandwidth and the spectra of the multiterahertz pulses become narrower [see Fig. 5(b) and (c)]. A notable reduction of the bandwidth occurs for $L \geq$ $580 \mu \mathrm{m}$ when the bandwidth drops by a factor of two. Nevertheless, even for $L=1050 \mu \mathrm{m}$, a sizeable FWHM bandwidth of $6.5 \mathrm{THz}$ can be achieved.

\section{NONPERTURBATIVE RESPONSE OF BULK SEMICONDUCTORS}

\section{A. Four-Wave Mixing Far Away From Interband Resonances}

In the coherent limit of light-matter interaction, the electronic system can be driven into the regime of Rabi flopping [32]. In this case, the polarization response cannot be described in frames of perturbative NL optics. This nonperturbative regime results in many intriguing phenomena such as self-induced transparency, quantum coherence, and high-harmonic generation [33]-[35]. Four-wave mixing (FWM) spectroscopy is particularly useful for probing the nonperturbative regime of quantum Rabi flopping since it bears information about the coherence response of a system.

Here, we study the NL polarization response of the interband resonance in the narrow-gap semiconductor $\mathrm{InSb}\left(\mathrm{E}_{g} / h=\right.$ $41 \mathrm{THz}$ ) at room temperature by means of FWM spectroscopy. The ultraintense terahertz pulses used in our experiment have a center frequency of $23 \mathrm{THz}$ which is located well below the direct band gap of InSb ensuring excitation far away from the interband resonance [see Fig. 6(a)]. An undoped (100)-oriented wafer of InSb has been mechanically polished to a thickness of $30 \mu \mathrm{m}$ and measured in a transmission geometry. The scheme of our terahertz multiwave mixing setup is shown in Fig. 6(b). A pair of terahertz pulses is produced by a terahertz Mach-Zehnder interferometer installed after the GaSe emitter crystal precluding 


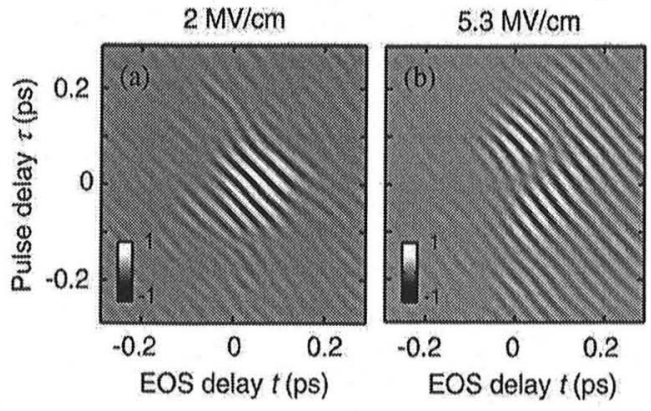

Fig. 7. Experimentally measured normalized four-wave mixing signal as a function of the EOS delay time $t$ and the delay between the excitation pulses $\tau$ for peak electric fields of (a) $2 \mathrm{MV} / \mathrm{cm}$ and (b) $5.3 \mathrm{MV} / \mathrm{cm}$

NL mixing effects of two beams within the emitter itself. We use a gold-coated germanium wafer placed at the Brewster angle as a broadband terahertz beam splitter. The transmitted pulse has variable delay with respect to the reflected pulse which additionally propagates through a second germanium wafer to match the phase dispersion. Both terahertz beams are tightly focused onto the sample and the transmitted transients are electrooptically detected with a 140- $\mu$ m-thick GaSe sensor. The transmitted field is detected as a function of the electrooptic sampling (EOS) delay time $t$ and the relative temporal offset between the terahertz pulses $\tau$. The NL signal is retrieved by subtracting the contributions of individually propagated pulses from the total response measured in the presence of both pulses. The FWM signal is extracted using a 2-D Fourier transform method [36]. In this way, contributions for noncoherent processes in InSb such as impact ionization [3] are filtered out.

Fig. 7(a) and (b) show the experimentally measured FWM signals for external peak terahertz electric fields of 2 and $5.3 \mathrm{MV} / \mathrm{cm}$, respectively. For the lower driving field, the FWM signal has an oval shape which is identical to the cross correlation function of both pulses [see Fig. 7(a)]. Such a response is expected in the case of a perturbative third-order nonlinearity and the shape of the FWM signal should not depend on the amplitude of the driving field. Surprisingly, the signal measured using the higher peak field of $5.3 \mathrm{MV} / \mathrm{cm}$ results in splitting of the FWM signal demonstrating a clear deviation from a simple perturbative response of InSb [see Fig. 7(b)]. The qualitative behavior observed in this experiment can be reproduced using a simplified model of a two-level system, representing the interband resonance in InSb. Our simulation is based on a semiclassical system of Maxwell-Bloch equations without the rotating wave approximation. Intriguingly, this simple two-level system model reproduces the splitting of the FWM signal observed in the experiment. A qualitative physical picture of this phenomenon can be understood by considering the polarization of the two-level system driven by an intensive terahertz field. Fig. 8(a) shows the electric field of both terahertz transients for $\tau=0 \mathrm{ps}$ which corresponds to the central part of the FWM signal where the observed splitting is the strongest. At early delay times when the field amplitude is below $3 \mathrm{MV} / \mathrm{cm}$, the response of the system is perturbative and the NL polarization $P_{\mathrm{NL}}$ follows the driving field as illustrated in Fig. 8(b). In the center of the driv-

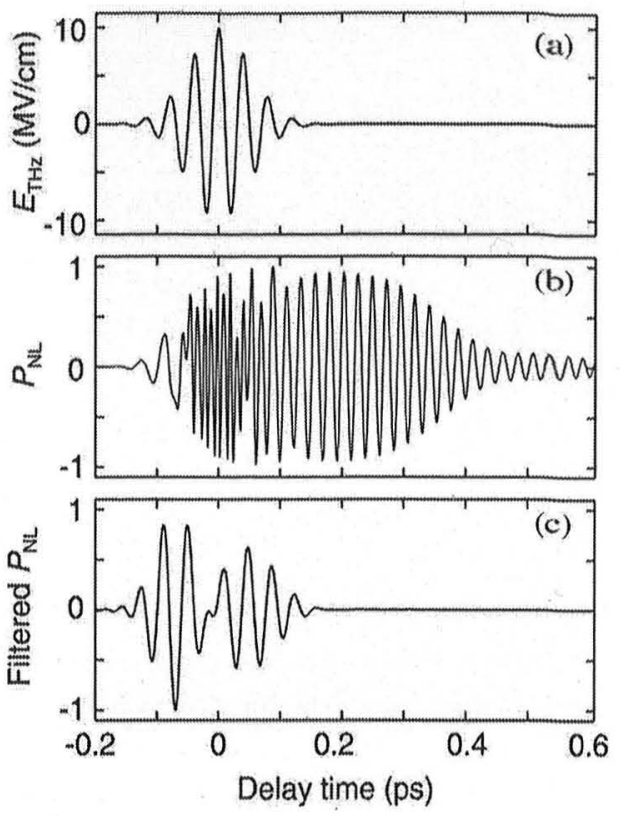

Fig. 8. Simulated nonperturbative response of a two-level system. (a) Driving electric field with an amplitude of $5 \mathrm{MV} / \mathrm{cm} /$ pulse for $\tau=0 \mathrm{ps}$. (b) Normalized total NL polarization $P_{\mathrm{NL}}$ of the system. (c) Part of the NL, polarization frequency filtered around the carrier frequency of the driving terahertz field and normalized to the maximum.

ing pulse where the total electric field reaches $10 \mathrm{MV} / \mathrm{cm}$, the generalized Rabi frequency becomes comparable to the detuning of the excitation pulse and the resonance frequency of the system. Therefore, in spite of a strongly nonresonant excitation, the system is driven into a nonperturbative regime of the carrier-wave Rabi flopping [32], [37]. In this regime, the major contribution to the polarization response around $t=0 \mathrm{ps}$ originates from higher harmonics [38], whereas the response at the fundamental frequency experiences a minimum as it is shown in Fig. 8(b) and (c). Finally, for $t>0.1 \mathrm{ps}$, the driving terahertz. field vanishes and the remnant polarization of the two-level system demonstrates a free induction decay characterized by the damped oscillation at the resonance frequency of $41 \mathrm{THz}$ [see Fig. 8(b)]. However, this signal is not observed in the FWM response which contains only polarization response around the carrier frequency of the driving terahertz field [see Fig. 8(c)].

Our study of the FWM response of bulk InSb provides clear evidence for the onset of a nonperturbative response to multiterahertz excitation. The extreme peak field amplitudes above 5 $\mathrm{MV} / \mathrm{cm}$ lead to Rabi flopping on a subpicosecond timescale with frequencies comparable to the excitation detuning of $18 \mathrm{THz}$ This extremely NL regime points out the high potential of the broadband high-field multiterahertz technology for coherent control of quantum states in bulk semiconductors and semiconductor nanostructures.

\section{B. Dynamical Franz-Keldysh Effect}

The Franz-Keldysh effect is the change in optical absorption of a semiconductor under the influence of an applied electric field [39]. It can be understood in terms of photon-assisted interband tunneling. Thus, the observation of a noticeable 


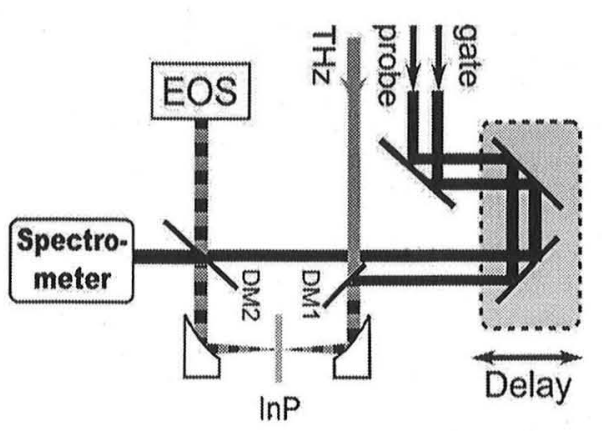

Fig. 9. Terahertz bias/near-infrared probe setup. The ultrashort broadband probe pulse is overlapped collinearly with the terahertz transient by a dichroic mirror (DM1), focused onto the InP sample and detected by a spectrometer. The terahertz beam is collimated, overlapped with the gate pulse on DM2 and detected via EOS.

electroabsorption requires the application of very high electric fields. However, accessible static electric fields are limited by the dielectric breakdown of a sample. This problem is avoided when an external field is applied dynamically on an ultrashort timescale. Therefore, single-cycle high-field terahertz transients represent a perfect tool for studies of the DFKE in bulk semiconductors under extremely intense fields well beyond the limit set by dielectric breakdown.

The terahertz bias/near-infrared probe setup developed for the study of the DFKE is schematically depicted in Fig. 9. The broadband 8 -fs-short probe and gating pulses are generated by two branches of the Er:fiber laser system as described in Section II and are synchronized with an extremely low timing jitter [40]. The central wavelength of the probe pulse is tuned close to the absorption edge of the studied InP sample located around $935 \mathrm{~nm}$. This experimental scheme allows us to detect simultaneously the inherently phase-locked terahertz. field and the field-induced changes in near-infrared absorption with a subcycle time resolution. The external terahertz peak electric field reaches $11 \mathrm{MV} / \mathrm{cm}$ which corresponds to fields of $5.4 \mathrm{MV} / \mathrm{cm}$ inside the InP sample.

The sample under study is a 230-nm-thick epitaxially grown InP layer deposited on a diamond substrate using a lift-off process. The (100)-orientation of the sample leads to a vanishing electrooptic effect. This allows us to avoid artifact signals due to the partially polarization sensitive elements like dichroic mirrors. The spectrally integrated relative transmission change $\Delta T / T$ of the InP sample measured at room temperature is shown in Fig. 10. Since a larger portion of the spectral weight of the probe pulse is located below the band gap energy, the signal is dominated by the field-induced absorption below the absorption edge and reaches its maximum value of almost $50 \%$ at the peak of the terahertz field around zero delay time [see Fig. 10(b)]. Remarkably, the subcycle time resolution of our experiment allows us to resolve a coherent modulation of the transmission change. A detailed analysis shows that the modulation occurs exactly at twice the carrier frequency of the driving terahertz field. Such a modulation in the time domain is equivalent to a sideband generation in the frequency domain predicted for the DFKE [41], [42]. Besides the modulation at the doubled frequency, one expects a series of even harmonics

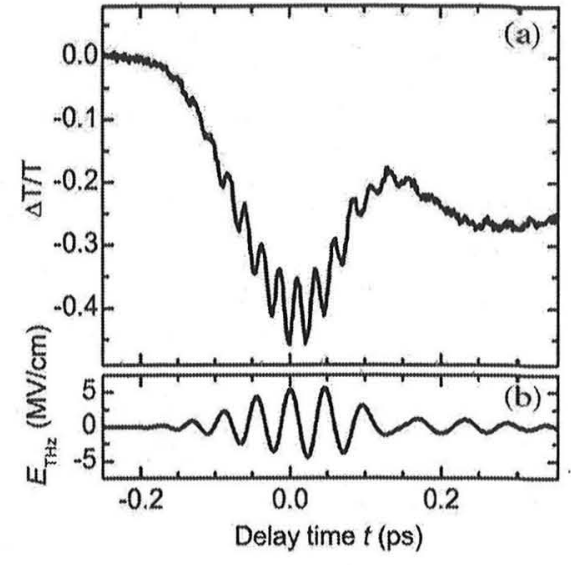

Fig. 10. (a) Spectrally integrated relative transmission change $\Delta T / T$ induced by a terahertz transient. (b) Temporal profile of the terahertz bias field.

sidebands [41]. However, our experimental spectra demonstrate only the second harmonic centered around $43 \mathrm{THz}$ and no clear modulation at $86 \mathrm{THz}$ which is probably too weak to be resolved. Another intriguing feature is a residual absorption at later delay times $t>0.2 \mathrm{ps}$ when the terahertz bias field vanishes [see Fig. 10(b)]. We attribute this absorption to the band gap renormalization by the high-density electron-hole plasma generated in InP by the intense terahertz bias pulses. The observed transmission decrease by $25 \%$ corresponds to a red-shift of the absorption edge of about $20 \mathrm{meV}$. An estimation based on the expression given in [43] results in an electron-hole density of $10^{17} \mathrm{~cm}^{-3}$. Our numerical modeling indicates that the interband Zener tunneling alone can provide the required plasma density.

\section{CONCLUSION}

We demonstrate that the DFG generation of intense terahertz radiation can be efficiently driven by using an OPA with type II phase matching for the amplification of the broadband signal pulses. This approach offers a number of advantages over type I phase matching: 1) reliable separation of the signal and the idler for a long wavelength operation, 2) negligible chirp of the signal pulse, 3) better stability of the terahertz pulses, and 4) generation of few-cycle terahertz transients with peak electric fields up to $25 \mathrm{MV} / \mathrm{cm}$. At the same time, the method also allows generation of ultrabroadband single-cycle multiterahertz transients with amplitudes up to $10 \mathrm{MV} / \mathrm{cm}$. The high time resolution provided by the NL fiber technology and the excellent phase stability of the terahertz transients pave the way toward previously unattainable information about high-field phenomena on a subcycle timescale.

As examples for the capabilities given by our system, we demonstrate field-resolved terahertz FWM spectroscopy of bulk InSb which provides evidence for a nonperturbative response that is reached even in the regime of the excitation far from the interband resonance. The combination of exceptionally intense terahertz pulses with the high stability and sensitivity of our setup enables multiwave mixing experiments in a large variety of condensed matter systems which demonstrate resonances in the 
multiterahertz spectral range. Particularly interesting candidates are a photon echo spectroscopy of hydrogen-bonded liquids [44] and a coherent control of the order parameter in superconductors [45], [46].

In addition, the DFKE in the bulk semiconductor InP has been resolved on the subcycle timescale. The developed experimental scheme is promising for investigations of extreme nonequilibrium transport in bulk semiconductors such as velocity overshoot [47] and Bloch oscillations [48].

\section{ACKNOWLEDGMENT}

The authors would like to thank S. Mährlein for the help in experiments, as well as A. Sell, M. Marangoni, and G. Cerullo for useful discussions and collaboration in the development of the high-field terahertz system.

\section{REFERENCES}

[1] L. Razzari, F. H. Su, G. Sharma, F. Blanchard, A. Ayesheshim, H.-C Bandulet, R. Morandotti, J.-C. Kieffer, T. Ozaki, M. Reid, and F. A. Hegmann, "Nonlinear ultrafast modulation of the optical absorption of intense few-cycle terahertz pulses in n-doped semiconductors," Phys. Rev. B, vol. 79, p. 193204, 2009.

[2] W. Kuehn, P. Gaal., K. Reimann, M. Woerner, T. Elsaesser, and R. Hey, "Coherent ballistic motion of electrons in a periodic potential," Phys. Rev. Let1., vol. 104, p. 1466()2, 2010.

[3] M. C. Hoffmann, J. Hebling, H. Y. Hwang, K.-L. Yeh, and K. A. Nelson, "Impact ionization in InSb probed by terahertz pump terahertz probe spectroscopy," Phys. Rev. B, vol. 79, p. 161201, 2009.

[4] H. Hirori, K. Shinokita, M. Shirai, S. Tani, Y. Kadoya, and K. Tanaka, "Extraordinary cartier multiplication gated by a picosecond electric field pulse," Nature Commun., vol. 2, p. 594, 2011.

[5] C. W.Luo, K. Reimann, M. Woerner, T. Elsaesser, R. Hey, and K. H. Ploog, "Phase-resolved nonlinear response of a two-dimensional electron gas under femtosecond intersubband excitation," Phys. Rev, Lett., vol. 92 p. 047402, 2004.

[6] S. Leinß, T. Kampfrath, K. v. Volkmann, M. Wolf, J. T. Steiner, M. Kira, S. W. Koch, A. Leitenstorfer, and R. Huber, "Terahertz coherent control of optically dark paraexcitons in $\mathrm{Cu}_{2} \mathrm{O}$," Phys. Rev. Lett, vol. 101,p. 246401 , 2008.

[7] A. Sell, A. Leitenstorfer, and R. Huber, "Phase-locked generation and field-resolved detection of widely tunable terahertz pulses with amplitudes exceeding $100 \mathrm{MV} / \mathrm{cm}$," Opt. Lett., vol. 33, no. 23, pp. 2767 ..2769, 2008.

[8] F. Junginger, A. Sell, O. Schubert, B. Mayer, D. Brida, M. Marangoni, G. Cerullo, A. Leitenstorfer, and R. Huber, "Single-cycle multiterahertz transients with peak fields above $10 \mathrm{MV} / \mathrm{cm}$," Opt. Lett., vol. 35, no. 15, pp. $2645 \cdots 2647,2010$.

[9] A. H. Chin, J. M. Bakker, and J. Kono, "Ultrafast electroabsorption at the transition between classical and quantum response," Phys. Rev. Lett. vol. 85, pp. 3293-3296, 2000.

[10] A. Srivastava, R. Srivastava, J. Wang, and J. Kono, "Laser-induced aboveband-gap transparency in GaAs," Phys. Rev. Lett., vol. 93, p. 157401, 2004.

[11] M. D. Thomson, M. Kress, T. Löffler, and H. G. Roskos, "Broadband THz emission from gas plasmas induced by femtosecond optical pulses: From fundamentals to applications," Laser Photon. Rev., vol. 1, pp. 349-368, 2007.

[12] J. Dai, J. Liu, and X.-C. Zhang, "Terahertz wave air photonics: Terahertz wave generation and detection with laser-induced gas plasma," IFEE Sel. Topics Quantum Electron., vol. 17, no. 1, pp. 183-190, Jan./Feb. 2011.

[1.3] A. Rice, Y. Jin, X. F. Ma, X. Zhang, D. Bliss, J. Larkin, and M. Alexander. "Terahertz optical rectification from (110) zinc-blende crystals," Appl. Phys. Lett., vol. 64, pp. 1324-1326, 1994

[14] R. A. Kaindl, D. C. Smith, M. Joschko, M. P. Hasselbeck, M. Woerner, and T. Elsaesser, "Femtosecond infrared pulses tunable from 9 to $18 \mu \mathrm{m}$ at an 88-MHz repetition rate," Opt. Lett., vol. 23, pp. 861 863. 1998.

[15] R. Huber, A. Brodschelm, F. Tauser, and A. Leitenstorfer, "Generation and field-resolved detection of femtosecond electromagnetic pulses tunable up to 41. THz," Appl. Pliys. Lett., vol. 76, no. 22, pp. 3191-31.93, 2000.
[16] A. Stepanov, J. Kuhl, I. Kozma, E. Riedle, and G. Almási, J. Hebling, "Scaling up the energy of $\mathrm{THz}$ pulses created by optical rectification," Opt. Express, vol. 13, pp. 5762-5768, 2005.

[17] J. Hebling, K.-L. Yeh. M. C. Hoffmann, B. Bartal, and K. A. Nelson, "Generation of high-power terahert $z$ pulses by tilted-pulse-front excitation and their application possibilities," J. Opt. Soc. Am. B, vol. 25, pp. B6 B19, 2008.

[18] H. Hirori, A. Doi, F. Blanchard, and K. Tanaka, "Single-cycle terahertz pulses with amplitudes exceeding $1 \mathrm{MV} / \mathrm{cm}$ generated by optical rectification in $\mathrm{LiNbO}_{3}$," Appl. Phys. Lett., vol. 98, no. 9, p. 091106, 2011.

[19] K.-L. Yeh, M. C. Hoffmann, J. Hebling, and K. A. Nelson, "Generation of $10 \mu \mathrm{J}$ ultrashort terahertz pulses by optical rectification," Appl. Phys. Lett., vol. 90, no. 17, p. 171121, 2007.

[20] A. G. Stepanov, L. Bonacina, S. V. Chekalin, and J..P. Wolf, "Generation of $30 \mu \mathrm{J}$ single-cycle terahertz pulses at $100 \mathrm{~Hz}$ repetition rate by optical rectification." Opt. Lett., vol. 33, no. 21, pp. 2497-2499, 2008.

[21] M. Jewariya, M. Nagai, and K. Tanaka, "Enhancement of terahertz wave generation by cascaded $\chi(2)$ processes in $\mathrm{LiNbO}_{3}, " \mathrm{~J}$. Opt. Soc. Am. B, vol. 26, no. 9, pp. A101 …A1()6, 2009.

[22] P. Hamm, C. Lauterwasser, and W. Zinth, "Generation of tunable subpicosecond light pulses in the midinfrared between 4.5 and $11.5 \mu \mathrm{m}$," Opt. Lett., vol. 18, no. 22, pp. 1943-1945, 1993.

[23] K. L. Vodopyanov and L. A. Kulevskii, "New dispersion relationships for GaSe in the $0.65 .18 \mu \mathrm{m}$ spectral region," Opt. Commun., vol. 118 , pp. $375-378,1995$.

[24] S. Ehret and H. Schneider, "Generation of subpicosecond infrared pulses tunable between $5.2 \mu \mathrm{m}$ and $18 \mu \mathrm{m}$ at a repetition rate of $76 \mathrm{MHz}$," Appl. Phys. B: Lasers Opt., vol. 66, pp. 27...30, 1998.

[25] K. Reimann, R. P. Smith, A. M. Weiner, T. Elsaesser, and M. Woerner, "Direct field-resolved detection of terahertz transients with amplitudes of megavolts per centimeter," Opt. Lett., vol. 28, pp. 471-473, 2003.

[26] D. Brida, C. Manzoni, G. Cirmi, M. Marangoni, S. Bonora, P. Villoresi, S. D. Silvestri, and G. Cerullo, "Few-optical-cycle pulses tunable from the visible to the mid-infrared by optical parametric amplifiers," J. Opt., vol. 12, no. 1, p. 013001, 2010.

[27] A. Baltuška, T. Fuji, and T. Kobayashi, "Controlling the carrier-envelope phase of ultrashort light pulses with optical parametric amplifiers," Phys. Rev. Lett., vol. 88, p. 133901, 2002.

[28] C. Manzoni, G. Cerullo, and S. D. Silvestri, "Ultrabroadband self-phase" stabilized pulses by difference-frequency generation," Opt. Lett., vol. 29 , no. 22 , pp. $2668-2670,2004$.

[29] A. Sell, G. Krauss, R. Scheu, R. Huber, and A. Leitenstorfer, " 8 -f's pulses from a compact Er:fiber system: Quantitative modeling and experimental implementation," Opt. Express, vol. 17, no. 2. pp. 1070 1.077, 2009.

[30] M. Nisoli, S. Stagira, S. D. Silvestri, O. Svelto, G. Valiulis, and A. Varanavicius, "Parametric generation of high-energy 14.5-fs light pulses at $1.5 \mu \mathrm{m}$," Opt. Lett., vol. 23, no. 8, pp. 630.632, 1998.

[31] R. Trebino, Frequency-Resolved Optical Gating: The Measurement of Ultrashort Laser Pulses. New York: Springer, 2002.

[32] C. Cohen-Tannoudji, B. Diu, and F. Laloë, Quantum Mechanics. New York: Wiley, 1977.

[33] H. Giessen, A. Knorr, S. Haas, S. Koch, S. Linden, J. Kuhl, M. Hetterich, M. Grün, and C. Klingshirn, "Self-induced transmission on a free exciton resonance in a semiconductor," Phys. Rev. Lett., vol. 81, pp. 426()-4263, 1998.

[34] T. Tritschler, O. D. Mücke, M. Wegener, U. Morgner, and F. X. Kärtner, "Evidence for third-harmonic generation in disguise of second-harmonic generation in extreme nonlinear optics," Phys. Rev. Lett., vol. 90. p. $217404,2003$.

[35] S. G. Carter, V. Birkedal, C. S. Wang, L. A. Coldren, A. V. Maslov, D. S. Citrin, and M. S. Sherwin, "Quantum coherence in an optical modulator," Science, vol. 310, pp. 651-653, 2005.

[36] W. Kuehn, K. Reimann, M. Woerner, and T. Elsaesser, "Phase-resolved two-dimensional spectroscopy based on collinear n-wave mixing in the ultrafast time domain," J. Chem. Phys., vol. 130, p. 164503, 2009.

[37] O. D. Mücke, T. Tritschler, M. Wegener, U. Morgner, and F. X. Kärtner, "Signatures of carrier-wave Rabi flopping in GaAs," Phys. Rev, Lett. vol. 87, p. (057401, 2001.

[38] T. Tritschler, O. D. Mücke, and M. Wegener, "Extreme nonlinear optics of two-level systems," Plys. Rev. A, vol. 68, p. (333404, 2003.

[39] H. Haug and S. W. Koch, Quantum Theory of the Optical and Electronic Properties of Semiconductors. 5thed. Singapore: World Scientific, 2009

[40] F. Adler, A. Sell, F. Sotier, R. Huber, and A. Leitenstorfer, "Attosecond relative timing jitter and $13 \mathrm{fs}$ tunable pulses from a two-branch Er: Fiber laser," Opt. Lett., vol. 32, no. 24, pp. 3504 3506, 2007. 
[41] Y. Yacoby, "High-frequency Franz-Keldysh effect," Phys. Rev., vol. 169, pp. $610.619,1968$.

[42] A. P. Jauho and K. Johnsen, "Dynamical Franz-Keldysh effect," Phys. Rev: Lett. vol. 76, pp. 4576 4579, 1996.

[43] P. Vashishta and R. K. Kalia, "Universal behavior of exchange-correlation energy in electron-hole liquid," Phys. Rev. B, vol. 25, pp. 6492 6495, 1982.

[44] M. L. Cowan, B. D. Bruner, N. Huse, J. R. Dwyer, B. Chugh, E. T. J. Nibbering, T. Elsaesser, and R. J. D. Miller, "Ultrafast memory loss and energy redistribution in the hydrogen bond network of liquid $\mathrm{H}_{2} \mathrm{O}$," Nature, vol. 434, pp. 199.202, 2005.

[45] T. Papenkort, T. Kuhn, and V. M. Axt, "Coherent control of the gap dynamics of BCS superconductors in the nonadiabatic regime," Phys. Rev. B, vol. 78, no. 13, p. 132505, 2008

[46] D. Fausti, R. I. Tobey, N. Dean, S. Kaiser, A. Dienst, M. C. Hoffmann, S. Pyon, T. Takayama, H. Takagi, and A. Cavalleri, "Light-induced superconductivity in a stripe-ordered cuprate," Science, vol. 331, no. 6014, pp. 189-191., 2011.

[47] A. Leitenstorfer, S. Hunsche, J. Shah, M. C. Nuss, and W. H. Knox, "Femtosecond charge transport in polar semiconductors," Phys. Rev. Lett., vol. 82, pp. 5140 5143,1999

[48] M. Wegener, Extreme Nonlinear Optics, 1st ed. New York: Springer, 2004. 